\title{
Ascertaining the Dynamic Competition in Channel Relationship Management
}

\author{
Lichung Jen ${ }^{1} \&$ Han-Kuang Tien ${ }^{1}$ \\ ${ }^{1}$ Department of International Business, National Taiwan University, Taipei, Taiwan \\ Correspondence: Han-Kuang Tien, College of Management, National Taiwan University, 1, Sec. 4, Roosevelt \\ Rd. Taipei 116, Taiwan. Tel: 886-2-3366-4983. E-mail: d95724001@ntu.edu.tw
}

Received: February 25, 2013 Accepted: March 10, 2013 Online Published: April 15, 2013

doi:10.5539/ijms.v5n3p36 URL: http://dx.doi.org/10.5539/ijms.v5n3p36

\begin{abstract}
One of the most important objectives of channel relationship management concerns how to effectively monitor the changes in each competitor's performance in different types of channel structures in order to grasp the dynamic state of competition. Unfortunately, competitor performance data is usually unavailable from a company's management information system. Under the condition of incomplete information, firms combine market share-related data from both internal and external sources and make predictions based on statistical models to ensure that they produce accurate market share information. In this paper, we propose a Bayesian model to improve the accuracy of market forecasts and thereby raise the quality of channel relationship management and help managers formulate more appropriate marketing strategies.
\end{abstract}

Keywords: channel relationship management, channel dependence matrix, market share, Bayesian model, IT industry

\section{Introduction}

Considering the current business environment, manufacturers are likely to face intense competition within marketing channels in the near future (Helander \& Moller, 2008; Hsieh \& Chou, 2011). One of the major challenges that confront manufacturers is how to both improve their awareness of the dynamic state of competition within these channels and monitor the structural changes in each manufacturer-specific channel in order to strengthen their own channel competitiveness. As such, manufacturers urgently need to assess their own performance across channels using market share information related to channels, which is the most widely accepted indicator of channel management performance (Dickson, 1983; Reibstein \& Farris, 1995; Zhou, Zhuang, \& Yip, 2007). Only when manufacturers obtain the market share information for their own specific channel structure can they possess the foresight to formulate an effective competitive strategy or marketing tactics. Since the use of market share information determines the marketing strategy direction, following incorrect market forecasts will lead to serious strategic mistakes. Therefore, marketers need to investigate how to obtain accurate market share information related to channels.

In general, market share information can be obtained from two major sources-internal and external sources. A manufacturer's internally established database can serve as a source of internal market information, but this information is usually limited to the manufacturer's own data; as such, it lacks perspective in terms of the overall market. In comparison, market information from external sources such as market surveys or market research companies (e.g., Nielsen, International Data Corporation [IDC]) can often be used to assess overall market conditions, but such information does not always accurately reflect the idiosyncrasies of a particular firm's market.

For these reasons, information from both major sources, internal and external, may be viewed as incomplete. To solve the subsequent problem of statistical inference, we require a statistical model that incorporates both aggregate (e.g., the overall channel structure's market share information as inferred from samples) and individual (e.g., the manufacturer's own internal sales data) information. Recently, Bayesian statistical analyses have been widely used in the context of various marketing research problems for this purpose (Allenby, Jen, \& Leone, 1996; Green \& Srinivasan, 1990; Jen, Chou, \& Allenby, 2009).

The current paper reviews the theoretical literature related to channel competition and proposes a Bayesian 
model with the intention of constructing a generalized statistical model of channel competition under incomplete information. The performance of the proposed model is robust in various market competition scenarios generated by simulation methods. Moreover, an empirical study based on the sales volume data of electronic notebook (hereafter notebook) products across different channels is conducted to demonstrate that the proposed model is superior in practice as well. Finally, general and managerial implications of the proposed model are discussed.

\section{Analysis of Channel Structure Competitiveness}

In order to set up an effective channel management strategy, Dickson (1983) proposed two major analytical frameworks: the distributor portfolio analysis (DPA) and the channel dependence matrix (CDM); the latter is also known as a relation matrix (see Table 1). This matrix reveals the market share for each manufacturer selling through any of the distributors in the target market. It shows the two-way dyadic relationship between manufacturers and distributors. The primary reason that channel market share has become a focus of research is that it represents the influence of manufacturers and distributors on the market (Coughlan, 2001; McCalley, 1992). Only when manufacturers can anticipate the market share associated with every channel's sales and accurately calculate the magnitude of their interdependence with each distributor can the so-called channel management be achieved.

Table 1. A channel dependence matrix - Market share analysis of channel structure

(a) An unbalanced market structure

\begin{tabular}{|c|c|c|c|c|c|c|c|}
\hline & & Distri & marke & & & & \\
\hline & & A & $\mathrm{B}$ & $\mathrm{C}$ & D & $\mathrm{E}$ & Total \\
\hline & $\mathrm{X}$ & $20 \%$ & $20 \%$ & $5 \%$ & $0 \%$ & $5 \%$ & $50 \%$ \\
\hline Manufacturer's & $\mathrm{Y}$ & $20 \%$ & $0 \%$ & $5 \%$ & $5 \%$ & $0 \%$ & $30 \%$ \\
\hline & Z & $10 \%$ & $0 \%$ & $0 \%$ & $5 \%$ & $5 \%$ & $20 \%$ \\
\hline & Total & $50 \%$ & $20 \%$ & $10 \%$ & $10 \%$ & $10 \%$ & $100 \%$ \\
\hline
\end{tabular}

(b) A balanced market structure

\begin{tabular}{lllllll}
\hline \multicolumn{7}{l}{ Distributor's market share } \\
\hline
\end{tabular}

Note: Adapted from "Distributor portfolio analysis and the channel dependence matrix: New techniques for understanding and managing the channel," by P. R. Dickson, 1983, Journal of Marketing, 47(3), p. 42.

In the relation matrix in Table 1(a), each cell represents the channel market share of a particular "manufacturer-distributor." The sum of each row represents each manufacturer's market share. The sum of each column represents each distributor's market share. For example, manufacturer $\mathrm{X}$ has a $50 \%$ market share, including $20 \%$ from distributor A, 20\% from distributor B, 5\% from distributor C, and 5\% from distributor $\mathrm{E}$. Thus, distributors A and B are the key channels currently controlled by manufacturer X. Further, since the supply sources for distributor B are highly concentrated, manufacturer $\mathrm{X}$ has a relatively greater control over distributor $\mathrm{B}$ than over distributor A. However, this does not ensure that the market will always be in a state of equilibrium. Supposing that there is an independent relationship between manufacturers and distributors, then distributor B will certainly seek to obtain supplies from manufacturers $\mathrm{Y}$ and $\mathrm{Z}$ in order to balance its competitive position relative to manufacturer $\mathrm{X}$. On the other hand, since manufacturer $\mathrm{X}$ enjoys enormous profits from distributor $\mathrm{B}$ owing to its advantageous position, it can provide distributor B with additional incentives to discourage it from developing more balanced channel relationships.

Table 1(b) shows balanced channel relationships under the so-called independent conditions. If a channel structure's market share is really as shown in Table 1(b), then the power of all manufacturers and distributors is in equilibrium. Unless new manufacturers and distributors enter or existing ones leave, causing a structural change in Table 1(b) and creating a state of disequilibrium, all firms will expend minimal costs to maintain the status quo in order to earn a normal profit. However, this phenomenon, which exists in theory, is not observed in 
practice. When market conditions are in a state of disequilibrium (see Table 1(a)), the firms in a relatively weak position (e.g., distributor B) will attempt to adjust the structure's development toward balanced channel relationships (as seen in Table 1(b)), while those in a relatively strong position (e.g., manufacturer X) will certainly adopt the necessary marketing tactics to secure their advantage and will lower their competitors' incentive to adjust the relationship toward that in Table 1(b).

The actions taken in the circumstances mentioned above are associated with the existence of perfect information across all parties. However, players in this game actually encounter information asymmetries when they consider potential strategic actions to influence the channel dependence matrix. The current study deals with this problem from the manufacturer's standpoint. One problem encountered by manufacturers during actual operations is that they often have a rough idea of marginal distribution (i.e., the sums of each row and column in Table 1), but they have no way of knowing the market share data for each channel (i.e., the values in each cell of Table 1). Without the market share data for each channel, manufacturers cannot determine the extent of their dependence on each distributor, thereby making channel management pointless.

The marketing problem that we investigate in this paper is how to estimate missing data under the condition of incomplete information. We adopt Bayesian statistical methods by combining market survey data provided by market research companies (prior information) as well as internal manufacturer data (observed data) to estimate the channel market share. Once the channel dependence matrix has been established, manufacturers can better understand market competition and thereby undertake strategic relationship marketing or develop strategic alliances with individual distributors.

\section{A Statistical Model of Channel Structure}

In order to combine internal and external market share information to better analyze competition within a channel structure under the condition of incomplete information, this paper begins by using a statistical theory to build a model for estimating the channel dependence matrix, as shown in Table 2 . We define $X_{i j}$ as the sales volume of the $i^{\text {th }}$ manufacturer through the $j^{\text {th }}$ distributor, $N$ as the total market sales volume, and $P_{i j}$ as the channel market share $\left(P_{i j}=X_{i j} / N\right)$.

Table 2. A channel dependence matrix - definition of variables

\begin{tabular}{|c|c|c|c|c|c|c|c|}
\hline & & \multicolumn{6}{|c|}{ Distributor's market share } \\
\hline \multirow{5}{*}{$\begin{array}{l}\text { Manufacturer's } \\
\text { market share }\end{array}$} & & $\mathrm{A}$ & $\mathrm{B}$ & $\mathrm{C}$ & D & E & Total \\
\hline & & $X_{I I}\left(P_{I I}\right)$ & $X_{12}\left(P_{12}\right)$ & $X_{13}\left(P_{13}\right)$ & $X_{14}\left(P_{14}\right)$ & $X_{15}\left(P_{15}\right)$ & $X_{1} \cdot\left(P_{l} \bullet\right.$ \\
\hline & Y & $X_{21}\left(P_{21}\right)$ & $X_{22}\left(P_{22}\right)$ & $X_{23}\left(P_{23}\right)$ & $X_{24}\left(P_{24}\right)$ & $X_{25}\left(P_{25}\right)$ & $X_{2} .\left(P_{2}.\right)$ \\
\hline & Z & $X_{31}\left(P_{31}\right)$ & $X_{32}\left(P_{32}\right)$ & $X_{33}\left(P_{33}\right)$ & $X_{34}\left(P_{34}\right)$ & $X_{35}\left(P_{35}\right)$ & $X_{3} \bullet\left(P_{3} \bullet\right)$ \\
\hline & Total & $X_{.1}(P .1)$ & $X_{.2}\left(P_{.2}\right)$ & $X_{.3}\left(P_{.3}\right)$ & $X_{\bullet 4}\left(P_{\bullet 4}\right)$ & $X_{.5}\left(P_{.5}\right)$ & $N\left(P_{\bullet \bullet}=1\right)$ \\
\hline
\end{tabular}

Based on the special characteristics of the random variable $X_{i j}$, we find that the structure of this matrix conforms to a multinominal distribution. In marketing modeling, a multinominal distribution is usually used to describe the purchasing choices of consumers with regard to a collection of brands (Lilien, Kotler, \& Moorthy, 1992). The function is of the following form:

$$
f(X \mid N, P)=\frac{N !}{\prod_{i} \prod_{j} X_{i j} !} \prod_{i} \prod_{j} P_{i j}^{X_{i j}} \quad \Sigma_{i} \Sigma_{j} X_{i j}=N, \quad 0 \leq X_{i j} \leq N, \quad \Sigma_{i} \Sigma_{j} P_{i j}=1, \quad 0 \leq P_{i j} \leq 1
$$

Regarding the problem of estimating the channel dependence matrix under the condition of incomplete information, the following conditions must be satisfied when designing a model. First, the sum of the estimated channel market share must be 1 . Second, the channel market share must possess the characteristic of dynamic change. Third, in the incomplete channel dependence matrix, a portion of the data is real data (e.g., the manufacturer's own sales volume); therefore, the sales volumes obtained by converting market share estimates must closely match the real data. Finally, the estimated market share must have a relatively small variance.

Suppose that, in the first period, manufacturers can receive complete market structure content (e.g., data obtained from detailed market surveys or purchased from specialized market research organizations), and in the second period, they can predict total market sales volume as well as receive partial information (the source may be the manufacturer's internal data or the marketer's estimates of several key cells in the channel dependence matrix). Then, marketers may face a challenge of how to update their original market share estimates on the basis of this new but incomplete information in order to develop a revised second-period channel dependence matrix, which 
can serve as a basis for predicting the third-period channel dependence matrix. Moreover, on the basis of this revised second-period channel dependence matrix, marketers can develop an appropriate channel strategy and improve channel management performance.

To solve the above problem, practitioners usually adopt the naive method of direct substitution to revise the values of the second-period estimated channel dependence matrix. In other words, when the first period's $P_{i j}(t)$ and the second period's $N(t+1)$ are defined, random draws $X_{i j}(t+1)$ can be generated on the basis of simulations of the multinominal distribution. These $X_{i j}(t+1)$ values are the second-period sales volume estimates of the $i^{\text {th }}$ manufacturer through the $j^{\text {th }}$ distributor. However, when second-period $X_{i j}(t+1)$ values are observed, one can substitute the actual values for predicted values, recalculate $N(t+1)$, and then update $P_{i j}(t+$ 1).

Practically, such an intuitive method has the advantage of being simple and easy to carry out; however, it disregards the conditions of statistical theory and characteristics of market structure. The primary shortcoming of such a direct substitution method is that it neglects the above-listed conditions for a model design. When researchers substitute the observed $X_{i j}(t+1)$ values for the original $X_{i j}(t+1)$ estimates, they overlook the structural characteristics of the first-period channel dependence matrix and implicitly assume that the channel dependence matrices of the two consecutive periods are mutually unrelated. In addition, the direct substitution method does not take into account the fact that all cells within the structure mutually influence each other.

To combine the original estimated values of the second-period channel dependence matrix (estimated on the basis of the structural characteristics of the first-period channel dependence matrix) and the observed incomplete information (representing actual possible structural changes in the second-period channel dependence matrix), this paper uses the rigorous derivation procedure of Bayesian statistical theory to organize information from two different kinds of sources into a common statistical distribution. In this way, we seek to simultaneously confront the strategic implications and obtain statistically predicted values with higher prediction accuracy. In terms of marketing practice, the significance of incorporating different kinds of data under a common statistical model is that all related analyses, estimations, and predictions in the same product market environment are carried out under the same conditions.

In the Bayesian approach, original information is treated as "prior knowledge," and the role of new information is referred to as "sample knowledge." We can obtain a posterior distribution by proportionally combining the prior probability and the likelihood function. This study treats the channel dependence matrix of the second period under incomplete information as sample knowledge and lets the random variable $X_{i j}(t+1)$ follow a multinominal distribution. Its likelihood function is like that shown in equation 1 . The parameters of this likelihood function are $P_{i j}(t+1)$ and $N(t+1)$.

In order for the prior distribution for $P_{i j}(t+1)$ to suit the form of the multinominal distribution function, its natural conjugate distribution should be a Dirichlet distribution (Berger, 1985). The Dirichlet distribution is a generalized gamma distribution; its probability density function is shown below (Johnson \& Kotz, 1972):

$$
D\left(P_{i j} \mid \alpha_{i j}\right)=\frac{\Gamma\left(\alpha_{0}\right)}{\prod_{i} \prod_{j} \Gamma\left(\alpha_{i j}\right)} \prod_{i} \prod_{j} P_{i j}^{\left(\alpha_{i j}-1\right)} \quad \Sigma_{i} \Sigma_{j} \alpha_{i j}=\alpha_{0}, \quad 0 \leq \alpha_{i j} \leq \alpha_{0}, \quad \Sigma_{i} \Sigma_{j} P_{i j}=1, \quad 0 \leq P_{i j} \leq 1
$$

where $\quad 0$ represents the total market sales volume (i.e., $N(t+1)$ ) and $\alpha_{i j}$ represents the sales volume of the $i^{\text {th }}$ manufacturer through the $j^{\text {th }}$ distributor (i.e., the predicted value $X_{i j}(t+1)$ obtained by taking the structural characteristics of the first-period channel dependence matrix as a basis). When the prior distribution of the market share variable follows a Dirichlet distribution (i.e., $P_{i j} \sim \mathrm{D}\left(\alpha_{i j}\right)$ ), and the sample, a multinominal distribution (i.e., $X_{i j} \sim \mathrm{M}\left(N, P_{i j}\right)$ ), the posterior distribution combining these two different information sources is as follows:

$$
\pi\left(P_{i j} \mid \alpha_{i j}, X_{i j}\right) \propto\left[\frac{\Gamma\left(\alpha_{0}\right)}{\prod_{i} \prod_{j} \Gamma\left(\alpha_{i j}\right)} \prod_{i} \prod_{j} P_{i j}^{\left(\alpha_{i j}-1\right)}\right] \cdot\left[\frac{N !}{\prod_{i} \prod_{j} X_{i j} !} \prod_{i} \prod_{j} P_{i j}^{X_{i j}}\right] \propto \prod_{i} \prod_{j} P_{i j}^{\left(\alpha_{i j}+X_{i j}-1\right)}
$$

According to the Bayesian theorem, we know that the posterior distribution of the channel market share $P_{i j}$ still follows a Dirichlet distribution (i.e., $P_{i j} \sim \mathrm{D}\left(\alpha_{i j}+X_{i j}\right)$ ).

To handle the estimation problem in equations $1-3$ of the model, this paper uses a Markov chain Monte Carlo estimation algorithm, also known as Gibbs sampling. It is an estimation procedure based on simulation (Gelfand \& Smith, 1990). On the basis of the conditional probability distributions of equations $1-3$, recursive simulation methods are used to generate random draws, and the values of these random draws serve as the conditional 
values of each conditional probability distribution. The process is then reiterated to generate the random draws for the next stage. After carrying out numerous simulations in this way, the convergence results yield random draws that are the best estimates of the parameters.

\section{Simulation Analysis}

To study the feasibility of the proposed model, we use simulated data and investigate the results under various scenarios. We assume that a product market is composed of three manufacturers $(\mathrm{X}, \mathrm{Y}, \mathrm{Z})$ and three distributors $(\mathrm{A}, \mathrm{B}, \mathrm{C})$; the initial channel dependence matrix is shown in Table 3. In this period, the total market sales volume is 1000 units. Of this total, manufacturer Y's $40 \%$ market share through distributor B is the largest, followed by manufacturer Z's 20\% market share through distributor C. Manufacturer X and distributor A are in relatively weak positions in this product market structure. Suppose that at the beginning of a period, manufacturer $\mathrm{X}$ obtains complete channel dependence matrix information by means of a large-scale market survey. However, the results from the survey would not necessarily coincide with the company's internal sales data. Further, for subsequent periods, manufacturer $\mathrm{X}$ would only know its own actual sales volume in each channel and have incomplete, speculative information about its competitors' sales volumes in each channel. As such, under the condition of incomplete information, manufacturer $\mathrm{X}$ would face a channel management problem of how to incorporate newly acquired sales data and channel dependence matrix information from the previous period in order to predict the channel dependence matrix for the next period. This is the only way that manufacturer $\mathrm{X}$ can formulate the most appropriate channel competition strategy and quickly respond to changes in the channel structure.

Table 3. Channel dependence matrix at the beginning stage

\begin{tabular}{|c|c|c|c|c|c|}
\hline \multicolumn{6}{|c|}{ Distributor's sales volume (market share) } \\
\hline & & A & $\mathrm{B}$ & $\mathrm{C}$ & Total \\
\hline Manufacturer's & $\mathrm{X}$ & $50(5 \%)$ & $100(10 \%)$ & $50(5 \%)$ & $200(20 \%)$ \\
\hline sales volume & Y & $20(2 \%)$ & $400(40 \%)$ & $60(6 \%)$ & $480(48 \%)$ \\
\hline (market share) & $\mathrm{Z}$ & $40(4 \%)$ & $80(8 \%)$ & $200(20 \%)$ & $320(32 \%)$ \\
\hline & Total & $110(11 \%)$ & $580(58 \%)$ & $320(32 \%)$ & $1000(100 \%)$ \\
\hline
\end{tabular}

Since predictions of total market sales volume are independent of the issues explored in this study, we regard them as exogenous variables and do not analyze them in detail. Suppose that we know from an appropriate aggregate prediction model that the next period's total market sales volume will grow by $10 \%$, that is, $N(t+1)=$ 1100 units. On the basis of the structure of the first period's channel dependence matrix, the prediction results for the second-period channel dependence matrix are shown in Table 4. The implication of these results is that the growth of the total market sales volume is uniformly spread over each "manufacturer-distributor" structure. Since new information has yet to be obtained, the results in Table 4 constitute the best possible prediction at this stage of strategy formation.

Table 4. Direct prediction for the $2^{\text {nd }}$ period of the channel dependence matrix

\begin{tabular}{|c|c|c|c|c|c|}
\hline \multicolumn{6}{|c|}{ Distributor's sales volume (market share) } \\
\hline & & A & B & $\mathrm{C}$ & Total \\
\hline Manufacturer's & $\mathrm{X}$ & $55(5 \%)$ & $110(10 \%)$ & $55(5 \%)$ & $220(20 \%)$ \\
\hline sales volume & $\mathrm{Y}$ & $22(2 \%)$ & $440(40 \%)$ & $66(6 \%)$ & $528(48 \%)$ \\
\hline (market share) & Z & $44(4 \%)$ & $88(8 \%)$ & $220(20 \%)$ & $352(32 \%)$ \\
\hline & Total & $121(11 \%)$ & $638(58 \%)$ & $352(32 \%)$ & $1100(100 \%)$ \\
\hline
\end{tabular}

Toward the end of the second period of strategy formation, however, manufacturer $\mathrm{X}$ already knows its own actual sales in channels A, B, and C and has obtained partial information about its competitors (the case wherein the manufacturer possesses no competitor information is considered in section 5). Manufacturers can therefore take advantage of this new but incomplete information to revise Table 4 and then use these revised results as a basis for predicting the third period's channel dependence matrix. Suppose that at the end of the second period, manufacturer X obtains its own actual sales data of 54, 118, and 56 units for distributors $\mathrm{A}, \mathrm{B}$, and $\mathrm{C}$, respectively. At the same time, manufacturer X learns that manufacturer Y's sales volume for distributor A is 24 units, and manufacturer Z's sales volume for distributor B is 92 units. The channel market share estimates of the 
revised channel dependence matrix, predicted according to the direct substitution method and the Bayesian model, are shown in Table 5.

Table 5. Revised prediction for the $2^{\text {nd }}$ period of the channel dependence matrix (standard error and posterior standard deviation)

\begin{tabular}{|c|c|c|c|c|c|c|c|}
\hline & & \multicolumn{3}{|c|}{ Direct substitution method } & \multicolumn{2}{|c|}{ Bayesian model } & \\
\hline \multirow{7}{*}{ Manufacturer } & & Distributor & & & Distributor & & \\
\hline & & A & B & $\mathrm{C}$ & A & B & $\mathrm{C}$ \\
\hline & $\mathrm{X}$ & 0.0483 & 0.1056 & 0.0501 & 0.0486 & 0.1062 & 0.0504 \\
\hline & & 0.0215 & 0.3951 & 0.0597 & 0.0216 & 0.3948 & 0.0593 \\
\hline & $\mathrm{Y}$ & $(0.000281)$ & $(0.012607)$ & $(0.007171)$ & $(0.000330)$ & $(0.006434)$ & $(0.003649)$ \\
\hline & 7 & 0.0394 & 0.0823 & 0.1980 & 0.0392 & 0.0828 & 0.1972 \\
\hline & & $(0.005822)$ & $(0.001069)$ & $(0.011221)$ & $(0.002974)$ & $(0.000584)$ & $(0.005548)$ \\
\hline
\end{tabular}

To compare the performance of these two methods, the variances of the estimates are first taken as the evaluation criteria. If an estimation method has a smaller variance such that the degree of fluctuation in parameter estimates is relatively small, then it is relatively efficient. According to the estimate results in Table 5, we find that the standard errors of the Bayesian method are smaller than those of the direct substitution method.

The second method for judging the model's accuracy is to compare the degree of discrepancy between the estimated sales volume and known sales data. To generate sales volume estimates, we use the market share estimates in Table 5 as the parameters in a multinominal distribution and randomly generate 500 simulated sales volumes. We also compare these values with an incomplete market matrix using the root mean square error (RMSE), the mean absolute deviation (MAD), and the mean absolute percentage error (MAPE).

Table 6 shows the channel dependence matrix of the sales volume predictions. Since both the direct substitution method and the Bayesian model have the capability of updating total market sales volumes, they yield the values of 1117 and 1111, respectively. It is worth noting that both of these revised predictions are higher than the predicted value for total market sales volume from the beginning of the period (1100). The reason for this is that observed sales volumes are all higher than previously predicted (e.g., the predicted value of $X_{12}$ was 110 , but the newly observed value is 118). Thus, the direct substitution method takes these known sales volumes and directly substitutes them for the original predicted values, causing the updated total market sales volume to increase to 1117 units. However, this method does not consider structural characteristics (i.e., fluctuations in market share within the channel dependence matrix); as such, this predicted value might be overestimated.

Table 6. Sales forecasting in the channel dependence matrix

\begin{tabular}{|c|c|c|c|c|c|c|c|}
\hline & & \multicolumn{3}{|c|}{ Direct substitution method } & \multicolumn{3}{|c|}{ Bayesian model } \\
\hline & \multicolumn{3}{|c|}{ Distributor } & \multirow[b]{2}{*}{$\mathrm{C}$} & \multicolumn{2}{|c|}{ Distributor } & \multirow[b]{2}{*}{$\mathrm{C}$} \\
\hline & & A & B & & A & B & \\
\hline \multirow{3}{*}{ Manufacturer } & $\mathrm{X}$ & 47.280 & 118.652 & 56.790 & 48.280 & 118.560 & 56.730 \\
\hline & $\mathrm{Y}$ & 24.214 & 444.170 & 67.396 & 24.142 & 440.778 & 66.534 \\
\hline & Z & 44.202 & 92.252 & 222.044 & 43.966 & 92.334 & 219.676 \\
\hline
\end{tabular}

On the other hand, the value predicted by the Bayesian model lies between the initial predicted value for total market sales volume from the beginning of the period and the value predicted by the direct substitution method. This is because the Bayesian model not only includes new information but also reflects the structural characteristics of the original channel dependence matrix. In fact, all Bayesian models have this characteristic in common; the method of using weights to make predicted values lie between original predicted values and values obtained from new information has already been shown to be superior in the literature (Allenby \& Rossi, 1999; Jen et al., 2009).

To compare the differences between the predicted values in Table 6 and the actual values, we calculate the RMSE, MAD, and MAPE, which are shown in Table 7. The RMSEs of the values predicted by the direct substitution method and the Bayesian model are 3.0436 and 2.5960, respectively, showing that the predictive 
errors of the Bayesian model are lower. The prediction accuracy of the Bayesian model is $15 \%$ higher than that of the direct substitution method. The other two measures of prediction accuracy also yield similar results.

Table 7. Comparison of prediction accuracy

\begin{tabular}{llll}
\hline & RMSE & MAD & MAPE \\
\hline Direct substitution method & 3.0436 & 1.7256 & $3.11 \%$ \\
Bayesian model & 2.5960 & 1.4972 & $2.67 \%$ \\
Degree of improvement of the Bayesian model & $15 \%$ & $13 \%$ & $14 \%$ \\
\hline
\end{tabular}

\section{Sensitivity Analysis}

From the above results, we see that the Bayesian model is a relatively good method of prediction. However, the example above is only one possible situation among numerous market competition scenarios; therefore, this paper considers an additional nine different scenarios on the basis of possible real-world changes in market competition so as to carry out a sensitivity analysis. In the original scenario, the new information observed in the second period is the actual sales volumes that exceeded the original predicted values. If we consider every possibility of an increase or a decrease in this way, we get nine potential scenarios, which can be generally divided into two major cases. In the first case, manufacturers have only their own sales volume information. In the second case, in addition to their own data, manufacturers also have partial data about their competitors. Further, each case is subdivided into five different scenarios. Taking the first case as an example, in scenarios 1 and 4, the predicted market growth and data obtained in the second period indicate movement in the same direction; in scenarios 2 and 3, indicated movements are in opposite directions; and in scenario 5 movements are a mixed result of a partial increase and a partial decrease. These ten possible change scenarios are compiled in Table 8 . When the known data from the second period and earlier predictions of total market sales volume indicate movements in opposite directions, this calls for necessary changes in the known data cells. When indicated movements are in the same direction, the situation depends on whether or not the scope of the movement exceeds the original predictions. The detailed design of the scenarios is shown in Table 8 and the estimation results of each scenario using the Bayesian model are depicted in Table 9.

Table 8. Design of scenarios

\begin{tabular}{lll}
\hline & $\begin{array}{l}\text { Conditions of information known in the } 2^{\text {nd }} \\
\text { (incomplete channel dependence matrix) }\end{array}$ & $\begin{array}{l}\text { Conditions for total market sales } \\
\text { volume in the } 2^{\text {nd }} \text { period }\end{array}$ \\
\hline Knows one's own & 1. All known $X_{i j}(t+1)$ increase & $N(t+1)$ increase \\
sales volume and & 2. All known $X_{i j}(t+1)$ increase & $N(t+1)$ decrease \\
partial information & 3. All known $X_{i j}(t+1)$ decrease & $N(t+1)$ increase \\
of competitors' key & 4. All known $X_{i j}(t+1)$ decrease & $N(t+1)$ decrease \\
cells $X_{i j}(t+1)$ & 5. Some of known $X_{i j}(t+1)$ increase and the others $N(t+1)$ increase \\
& decrease & $N(t+1)$ increase \\
Knows only one's & 6. All known $X_{i j}(t+1)$ increase & $N(t+1)$ decrease \\
own sales volume & 7. All known $X_{i j}(t+1)$ increase & 9. All known $X_{i j}(t+1)$ decrease \\
$X_{i j}(t+1)$ & 10. Some of known $X_{i j}(t+1)$ increase and the others $N(t+1)$ increase \\
& decrease & $N(t+1)$ increase \\
\hline
\end{tabular}


Table 9. Estimation results for each scenario

\begin{tabular}{|c|c|c|c|c|c|c|c|c|c|c|c|}
\hline \multicolumn{2}{|l|}{$\gamma_{\text {CDM Cells }}$} & 1 & 2 & 3 & 4 & 5 & 6 & 7 & 8 & 9 & \\
\hline \multicolumn{2}{|l|}{ Market Scenario } & $X_{11}$ & $X_{21}$ & $X_{31}$ & $X_{12}$ & $X_{22}$ & $X_{32}$ & $X_{13}$ & $X_{23}$ & $X_{33}$ & Total \\
\hline \multicolumn{12}{|c|}{ Sales volume known $(\mathrm{t}+1)$} \\
\hline$X \uparrow N \uparrow$ & 1 & 54 & 24 & & 118 & & 92 & 56 & & & 1100 \\
\hline$X \uparrow N \downarrow$ & 2 & 54 & 24 & & 118 & & 92 & 56 & & & 900 \\
\hline$X \downarrow N \uparrow$ & 3 & 46 & 16 & & 93 & & 71 & 44 & & & 1100 \\
\hline$X \downarrow N \downarrow$ & 4 & 46 & 16 & & 93 & & 71 & 44 & & & 900 \\
\hline$X \uparrow \downarrow N \uparrow$ & 5 & 46 & 16 & & 118 & & 92 & 44 & & & 1100 \\
\hline$X \uparrow N \uparrow$ & 6 & 54 & & & 118 & & & 56 & & & 1100 \\
\hline$X \uparrow N \downarrow$ & 7 & 54 & & & 118 & & & 56 & & & 900 \\
\hline$X \downarrow N \uparrow$ & 8 & 46 & & & 93 & & & 44 & & & 1100 \\
\hline$X \downarrow N \downarrow$ & 9 & 46 & & & 93 & & & 44 & & & 900 \\
\hline$X \uparrow \downarrow N \uparrow$ & 10 & 46 & & & 118 & & & 44 & & & 1100 \\
\hline \multicolumn{12}{|c|}{ Estimated market share $(t+1)$} \\
\hline$X \uparrow N \uparrow$ & 1 & 0.0486 & 0.0216 & 0.0392 & 0.1062 & 0.3948 & 0.0828 & 0.0504 & 0.0593 & 0.1972 & 1.00 \\
\hline$X \uparrow N \downarrow$ & 2 & 0.0567 & 0.0252 & 0.0365 & 0.1239 & 0.3655 & 0.0966 & 0.0588 & 0.0545 & 0.1824 & 1.00 \\
\hline$X \downarrow N \uparrow$ & 3 & 0.0432 & 0.0150 & 0.0425 & 0.0874 & 0.4267 & 0.0667 & 0.0413 & 0.0640 & 0.2133 & 1.00 \\
\hline$X \downarrow N \downarrow$ & 4 & 0.0514 & 0.0178 & 0.0399 & 0.1039 & 0.3992 & 0.0793 & 0.0491 & 0.0599 & 0.1996 & 1.00 \\
\hline$X \uparrow \downarrow N \uparrow$ & 5 & 0.0421 & 0.0146 & 0.0405 & 0.1080 & 0.4062 & 0.0842 & 0.0402 & 0.0610 & 0.2032 & 1.00 \\
\hline$X \uparrow N \uparrow$ & 6 & 0.0487 & 0.0198 & 0.0395 & 0.1066 & 0.3970 & 0.0795 & 0.0505 & 0.0597 & 0.1987 & 1.00 \\
\hline$X \uparrow N \downarrow$ & 7 & 0.0579 & 0.0188 & 0.0377 & 0.1266 & 0.3781 & 0.0754 & 0.0601 & 0.0565 & 0.1888 & 1.00 \\
\hline$X \downarrow N \uparrow$ & 8 & 0.0425 & 0.0207 & 0.0414 & 0.0860 & 0.4154 & 0.0830 & 0.0407 & 0.0626 & 0.2078 & 1.00 \\
\hline$X \downarrow N \downarrow$ & 9 & 0.0508 & 0.0199 & 0.0399 & 0.1028 & 0.3989 & 0.0798 & 0.0486 & 0.0599 & 0.1993 & 1.00 \\
\hline$X \uparrow \downarrow N \uparrow$ & 10 & 0.0420 & 0.0202 & 0.0404 & 0.1077 & 0.4050 & 0.0809 & 0.0401 & 0.0610 & 0.2026 & 1.00 \\
\hline \multicolumn{12}{|c|}{ Estimated sales volume $(t+1)$} \\
\hline$X \uparrow N \uparrow$ & 1 & 54.00 & 23.97 & 43.54 & 118.07 & 438.86 & 92.04 & 56.00 & 65.92 & 219.20 & $1,111.61$ \\
\hline$X \uparrow N \downarrow$ & 2 & 54.00 & 23.97 & 34.79 & 118.08 & 348.21 & 92.04 & 56.00 & 51.91 & 173.74 & 952.74 \\
\hline$X \downarrow N \uparrow$ & 3 & 46.00 & 15.98 & 45.32 & 93.07 & 454.59 & 71.04 & 44.00 & 68.15 & 227.24 & $1,065.40$ \\
\hline$X \downarrow N \downarrow$ & 4 & 46.00 & 15.98 & 35.71 & 93.07 & 357.62 & 71.04 & 44.00 & 53.62 & 178.77 & 895.82 \\
\hline$X \uparrow \downarrow N \uparrow$ & 5 & 46.00 & 15.97 & 44.28 & 118.10 & 444.37 & 92.07 & 44.00 & 66.73 & 222.31 & $1,093.84$ \\
\hline$X \uparrow N \uparrow$ & 6 & 53.99 & 21.92 & 43.79 & 118.05 & 439.79 & 88.03 & 55.99 & 66.18 & 220.08 & $1,107.83$ \\
\hline$X \uparrow N \downarrow$ & 7 & 53.99 & 17.55 & 35.19 & 118.05 & 352.49 & 70.28 & 55.99 & 52.69 & 175.98 & 932.20 \\
\hline$X \downarrow N \uparrow$ & 8 & 45.99 & 22.33 & 44.83 & 93.04 & 449.24 & 89.74 & 43.99 & 67.66 & 224.73 & $1,081.55$ \\
\hline$X \downarrow N \downarrow$ & 9 & 45.99 & 18.02 & 36.06 & 93.04 & 360.88 & 72.23 & 43.99 & 54.19 & 180.30 & 904.71 \\
\hline$X \uparrow \downarrow N \uparrow$ & 10 & 45.99 & 22.15 & 44.30 & 118.07 & 443.77 & 88.68 & 43.99 & 66.88 & 221.98 & $1,095.81$ \\
\hline
\end{tabular}

The results of each scenario are described as follows:

Scenario 1 - All known $X_{i j}(t+1)$ increase and $N(t+1)$ increases. This is the case described above, so we do not explain it further here.

Scenario 2 - All known $X_{i j}(t+1)$ increase and $N(t+1)$ decreases. The size of the total market shrinks, but the sales volumes of the firm and of some of its competitors increase. From the estimates in Table 9, we note that for all those firms whose sales increased, the second-period market share is higher than it was in the first period (cells 1, 2, 4, 6, 7), while the market share falls for the other firms.

Scenario 3 - All known $X_{i j}(t+1)$ decrease and $N(t+1)$ increases. The size of the total market grows (as in the high season) but declines in some known cells. From Table 9, we know that for all those firms whose sales declined, the second-period market share $(4.3 \%, 1.5 \%, 8.7 \%, 6.7 \%, 4.13 \%)$ is lower than that of the first period $(5 \%, 2 \%, 10 \%, 8 \%, 5 \%)$. As such, the other firms take the opportunity to steal market share, increasing their overall market share.

Scenario 4 - All known $X_{i j}(t+1)$ decrease and $N(t+1)$ decreases. The market is predicted to decline, and the known data in the second period shows a decline (e.g., the sales of all firms are poor during the off-season). However, if the decline reflected in the known data does not exceed the predicted market decline, then the market share in these cells can rise (cells 1 and 4 rise from $5 \%$ and $10 \%$ to $5.1 \%$ and $10.34 \%$, respectively); 
otherwise, it falls.

Scenario 5 - Some of the known $X_{i j}(t+1)$ increase while the others decrease, and $N(t+1)$ increases. Overall, the market is in a growth stage. The known sales data of the second period includes both increasing and decreasing situations. Here, the known data assumes that a certain channel's market share is increasing (i.e., cells 4 and 6), while those of the remaining channels are decreasing (e.g., when a certain channel is on the rise and becomes a focus of competition, the resources and budget devoted to other channels are correspondingly reduced). Table 9 shows that the share in cell 4 increases from $10 \%$ to $10.8 \%$ and that in cell 6 increases from $8 \%$ to $8.42 \%$.

Scenario 6 - All known $X_{i j}(t+1)$ increase and $N(t+1)$ increases. The industry is growing and firms are working hard to develop new markets or further penetrate old markets. However, since competitors are taking a piece of the pie, manufacturer X has a relatively good record only with distributor B (from $10 \%$ to $10.6 \%$ ), while its results elsewhere differ little from the previous period, indicating that the competitive situation has not changed significantly.

Scenario 7 - All known $X_{i j}(t+1)$ increase and $N(t+1)$ decreases. The market is expected to decrease (e.g., gradually entering the last stage of the product life cycle). However, internal data from manufacturer $\mathrm{X}$ is growing (e.g., product repositioning causes the life cycle to be extended and competitors have difficulty following suit). Therefore, generally speaking, manufacturer X's market share in the second period is higher than that of the first period (cells $1,4,7$ ), indicating that a product repositioning strategy truly breathes new life into a company facing intense competition and a declining market.

Scenario 8 - All known $X_{i j}(t+1)$ decrease and $N(t+1)$ increases. The complete opposite of scenario 7 creates a situation in which the market shares of firms in the second period $(4.25 \%, 8.6 \%, 4.07 \%)$ are all lower than those in the first period $(5 \%, 10 \%, 5 \%)$, while the market status correspondingly drops precipitously. Each firm needs to quickly handle the crisis.

Scenario 9 - All known $X_{i j}(t+1)$ decrease and $N(t+1)$ decreases. The market is declining (e.g., a traditional sunset industry) and manufacturer X's market share is decreasing along with it, indicating a gradual onset of management difficulties. If the scope of the firm's decline is no larger than the degree of the overall slide in the market, then a harvest strategy should be adopted. However, if the magnitude of the firm's sales decline is greater than that of the overall slide in the market, then a divested strategy should be adopted.

Scenario 10 - Some of the known $X_{i j}(t+1)$ increase while the others decrease, and $N(t+1)$ increases. When the market is growing, manufacturer X's second period sales volumes in each channel are both increasing and decreasing: they increases through distributor B (from 10\% to 10.8\%), but decrease through distributors A and C (from $5 \%$ to $4.2 \%$, and $5 \%$ to $4.0 \%$, respectively). If manufacturer $\mathrm{X}$ seeks to establish a market position in distributor B, but neglects the other distributors, this would be an ineffective strategy.

To sum up the above results, businesses are not only concerned about their current situation, but also hope to predict the future. Combining complete market information from the previous period with known but incomplete information from the present period enables an estimation of the market position of each manufacturer in each channel (i.e., channel market share) in the current period. Essentially, there are a myriad of possible strategies that one's competitors might choose; as such, simulations of different scenarios rely on managers' understanding of the industry and the market. The importance of the current study rests on our rigorous theoretical framework, which provides firms with a feasible method of prediction.

\section{Empirical Study}

To empirically verify the proposed model, we apply it to real-world sales data from the information technology (IT) industry, which is one of the fastest growing industries in the world. This growth is spurred by its dynamic, fast-paced, innovative, and productive nature, and its impact runs well beyond the boundaries of the industry itself (Mendelson \& Whang, 2000). The key characteristic of the IT industry is its fast clockspeed caused by the fast pace of changes taking place in terms of product design, manufacturing processes, and organizational structure (Fine, 1999; Mendelson \& Pillai, 1998).

In addition, the more downstream the logistics chain - an end accesses to the customers - the more dynamic changes the firms face. Within the IT industry, the fast pace of change and intensified competition mean that all competitive advantages are temporary, especially for products such as personal computers and communication devices, which are characterized by high product values, short product life cycles, and high demand in terms of customization. As a result, IT manufacturer marketing managers must continuously update the market structure information to compete against their rivals. 
We use sales volume data of notebook manufacturers through different channels provided by a market research company in Taiwan as the empirical data. Quarterly data for the period 1995-2001 were selected as the calibrated data. The dataset consists of 17 manufacturers/brands, including major players such as IBM, Dell, HP, and Compaq. The channels consist of six distributors, including direct outbound, direct inbound, dealer/value added reseller/system integrator, retail, Internet direct, and others. Hence, a $17 \times 6$ channel dependence matrix for each quarter is formed for the following empirical analysis.

Because a significant amount of missing sales data is involved in constructing the channel dependence matrix, the proposed model is suitable for solving this problem. Taking the market sales volume in the $26^{\text {th }}$ and $27^{\text {th }}$ period as an example, the analyst encounters the problem of how to incorporate the incomplete $27^{\text {th }}$ period information with the previous period's data in order to estimate the complete structure of the $27^{\text {th }}$ period relation matrix. The estimated market share volume values of the revised relation matrix in the $27^{\text {th }}$ period predicted according to the direct substitution method and the Bayesian statistical model are separately shown in Table 10 .

Table 10. Market share prediction based on Bayesian statistical model for the $27^{\text {th }}$ period (\%)

\begin{tabular}{lllllll}
\hline Brands & Distributors & & & & & \\
Acer & 0.0015 & 4.6269 & 8.9311 & 5.3319 & 0.0015 & 0.0015 \\
Apple & 0.0016 & 0.0015 & 1.2759 & 1.2774 & 0.0016 & 0.0016 \\
AST & 0.0017 & 0.0016 & 0.0015 & 0.0015 & 0.0016 & 0.0015 \\
ASUS & 0.0015 & 0.0016 & 16.0251 & 4.2208 & 0.0016 & 0.0015 \\
Compaq & 0.0016 & 0.0016 & 8.6372 & 4.1608 & 0.4770 & 0.7114 \\
Dell & 0.0764 & 0.0904 & 0.3390 & 0.0016 & 0.0016 & 0.0015 \\
DTK & 0.0016 & 0.0016 & 0.0015 & 0.0016 & 0.0016 & 0.0016 \\
HP & 0.0016 & 0.0016 & 1.0594 & 0.1998 & 0.0015 & 0.0015 \\
IBM & 0.0016 & 0.0015 & 9.1173 & 4.1831 & 0.7415 & 0.0016 \\
LEO (FIC) & 0.0016 & 0.8327 & 3.6041 & 0.3726 & 0.0656 & 0.0212 \\
Mitac & 0.0016 & 0.0016 & 4.4675 & 0.8464 & 0.0016 & 0.0015 \\
NEC & 0.0015 & 0.0016 & 0.0016 & 0.0015 & 0.0016 & 0.0015 \\
Philips & 0.0016 & 0.0016 & 0.0016 & 0.0016 & 0.0016 & 0.0016 \\
Sharp & 0.0015 & 0.0015 & 0.0015 & 0.0016 & 0.0016 & 0.0016 \\
Tatung & 0.0016 & 0.0016 & 0.0016 & 0.0016 & 0.0016 & 0.0016 \\
Toshiba & 0.0015 & 0.4767 & 2.0169 & 0.6859 & 0.0015 & 0.0016 \\
TwinHead & 0.7721 & 1.9849 & 9.3989 & 2.8638 & 0.0016 & 0.0015 \\
\hline
\end{tabular}

The prediction results from Table 11 show that the RMSEs and MADs of the Bayesian statistical model are consistently lower than those of the direct substitution model for all periods. The predictability improvement of the Bayesian model over the direct substitution method is at least $50 \%$. Moreover, MAD - the other indicator of predictability—yields similar results. 
Table 11. Comparison of predictability of the notebook channel market

\begin{tabular}{|c|c|c|c|c|c|c|}
\hline \multirow[b]{2}{*}{ Period } & \multicolumn{2}{|c|}{ Direct substitution method } & \multicolumn{2}{|c|}{ Bayesian statistical method } & \multicolumn{2}{|c|}{$\begin{array}{l}\text { Degree of improvement } \\
\text { Bayesian statistical method }\end{array}$} \\
\hline & RMSE & MAD & RMSE & MAD & RMSE & MAD \\
\hline 2 & 0.0294 & 0.0097 & 0.0080 & 0.0024 & $73 \%$ & $75 \%$ \\
\hline 3 & 0.0279 & 0.0097 & 0.0059 & 0.0014 & $79 \%$ & $86 \%$ \\
\hline 4 & 0.0374 & 0.0097 & 0.0140 & 0.0038 & $63 \%$ & $61 \%$ \\
\hline 5 & 0.0395 & 0.0097 & 0.0077 & 0.0026 & $80 \%$ & $73 \%$ \\
\hline 6 & 0.0305 & 0.0097 & 0.0128 & 0.0034 & $58 \%$ & $65 \%$ \\
\hline 7 & 0.0259 & 0.0097 & 0.0106 & 0.0034 & $59 \%$ & $65 \%$ \\
\hline 8 & 0.0290 & 0.0097 & 0.0113 & 0.0038 & $61 \%$ & $61 \%$ \\
\hline 9 & 0.0278 & 0.0097 & 0.0045 & 0.0014 & $84 \%$ & $86 \%$ \\
\hline 10 & 0.0312 & 0.0098 & 0.0074 & 0.0022 & $76 \%$ & $77 \%$ \\
\hline 11 & 0.0348 & 0.0098 & 0.0095 & 0.0026 & $73 \%$ & $73 \%$ \\
\hline 12 & 0.0357 & 0.0098 & 0.0101 & 0.0031 & $72 \%$ & $69 \%$ \\
\hline 13 & 0.0346 & 0.0098 & 0.0057 & 0.0019 & $84 \%$ & $80 \%$ \\
\hline 14 & 0.0367 & 0.0098 & 0.0034 & 0.0009 & $91 \%$ & $91 \%$ \\
\hline 15 & 0.0369 & 0.0098 & 0.0056 & 0.0019 & $85 \%$ & $80 \%$ \\
\hline 16 & 0.0349 & 0.0098 & 0.0042 & 0.0011 & $88 \%$ & $88 \%$ \\
\hline 17 & 0.0304 & 0.0098 & 0.0071 & 0.0023 & $77 \%$ & $77 \%$ \\
\hline 18 & 0.0259 & 0.0098 & 0.0099 & 0.0031 & $62 \%$ & $68 \%$ \\
\hline 19 & 0.0261 & 0.0098 & 0.0079 & 0.0026 & $70 \%$ & $73 \%$ \\
\hline 20 & 0.0260 & 0.0098 & 0.0095 & 0.0030 & $64 \%$ & $69 \%$ \\
\hline 21 & 0.0265 & 0.0098 & 0.0096 & 0.0027 & $64 \%$ & $72 \%$ \\
\hline 22 & 0.0253 & 0.0098 & 0.0074 & 0.0027 & $71 \%$ & $72 \%$ \\
\hline 23 & 0.0244 & 0.0115 & 0.0077 & 0.0031 & $68 \%$ & $73 \%$ \\
\hline 24 & 0.0275 & 0.0121 & 0.0110 & 0.0029 & $60 \%$ & $76 \%$ \\
\hline 25 & 0.0265 & 0.0098 & 0.0133 & 0.0030 & $50 \%$ & $69 \%$ \\
\hline 26 & 0.0281 & 0.0098 & 0.0058 & 0.0021 & $79 \%$ & $78 \%$ \\
\hline 27 & 0.0266 & 0.0098 & 0.0044 & 0.0015 & $83 \%$ & $85 \%$ \\
\hline 28 & 0.0247 & 0.0115 & 0.0069 & 0.0025 & $72 \%$ & $78 \%$ \\
\hline
\end{tabular}

\section{Conclusions}

Two of the most important tasks pertaining to channel management are effective monitoring of changes in the channel dependence matrix and strengthening the competitiveness of key channels. In estimating the channel dependence matrix, the Bayesian model presented in this paper yields better results than the direct substitution method does, both in terms of estimation variance and prediction accuracy. At the same time, by means of a sensitivity analysis, we show that our Bayesian model can effectively revise the predicted results under a wide variety of scenarios. Furthermore, the superiority of the proposed model is also supported by our empirical study results.

Although this paper analyzes the competitive positions of manufacturers in distribution channels, in practice our model can also be used for other marketing topics. For example, its dimensions can be adjusted according to different decision problems pertaining to area, brand, customers, and design, among others. Furthermore, our model is not limited to two-dimensional market share analyses. When the number of dimensions is increased to three or more (e.g., manufacturer $\times$ channel $\times$ area), it is necessary to only redefine the random variable $X_{i j k}$ as $X_{m}$, where $m=1,2, \ldots, I \times J \times K$ and then carry out the analysis in sequence according to the assumptions of the multi-nominal distribution.

Future studies can develop competitive models based on the proposed model. For example, in an oligopoly market, the behavior between competitors is highly interactive (e.g., potential for collusion). We can take changes in the market share of each period as the dependent variables and the firms' marketing strategies (especially promotion strategies) as the independent variables to set up a hierarchical Bayesian model. In sum, marketing managers are forced to make some strategic decisions with incomplete information. The proposed 
model may shed some light on how to solve this issue in terms of channel management; as such, it deserves greater research attention in the future.

\section{References}

Allenby, G. M., \& Rossi, P. E. (1999). Marketing models of consumer heterogeneity. Journal of Econometrics, 89(1/2), 57-78. http://dx.doi.org/10.1016/S0304-4076(98)00055-4

Allenby, G. M., Arora, N., \& Ginter, J. L. (1998). On the heterogeneity of demand. Journal of Marketing Research, 35(3), 384-389.

Allenby, G. M., Jen, L., \& Leone, R. P. (1996). Economic trends and being trendy: The influence of consumer confidence on retail fashion sales. Journal of Business \& Economic Statistics, 14(1), 103-111.

Allenby, G. M., Leone, R. P., \& Jen, L. (1999). A dynamic model of purchase timing with application to direct marketing. Journal of American Statistics Association, 93(446), 365-374.

Berger, J. O. (1985). Statistical decision theory and Bayesian analysis. New York, NY: Springer-Verlag.

Coughlan, A. T. (2002). Marketing channels. Englewood Cliffs, NJ: Prentice Hall.

Dickson, P. R. (1983). Distributor portfolio analysis and the channel dependence matrix: New techniques for understanding and managing the channel. Journal of Marketing, 47(3), 35-44.

Fine, C. H. (1999). Clockspeed: Winning industry control in the age of temporary advantage. New York, NY: Perseus Publishing.

Gelfand, A. E., \& Smith, A. F. M. (1990). Sampling-based approaches to calculating marginal densities. Journal of American Statistics Association, 85(410), 398-409.

Green, P. E., \& Srinivasan, V. (1990). Conjoint analysis in marketing: New developments with implications for research and practice. The Journal of Marketing, 54(4), 3-19.

Helander, A., \& Moller, K. (2008). How to become solution provider: System supplier's strategic tools. Journal of Business-to-Business Marketing, 15(3), 247-89. http://dx.doi.org/10.1080/15470620802059265

Hsieh, M. H., \& Chou, W. C. (2010). Managing key account portfolios across the process of relationship development: A value proposition-desired value alignment perspective. Journal of Business-to-Business Marketing, 18, 83-119. http://dx.doi.org/10.1080/1051712X.2010.490932

Jen, L., \& Wang, S. J. (1998). Incorporating heterogeneity in customer valuation: An empirical study of health care direct marketing in Taiwan. International Journal of Operations and Quantitative Management, 4(3), 217-228.

Jen, L., Chou, C. H., \& Allenby, G. M. (2009). The importance of modeling temporal dependence of timing and quantity in direct marketing. Journal of Marketing Research, 46, 482-493.

Johnson, N. L., \& Kotz, S. (1972). Distributions in statistics: Continuous multivariate distributions. New York, NY: John Wiley.

Lilien, G. L., Kotler, P., \& Moorthy, K. S. (1992). Marketing models. Englewood Cliffs, NJ: Prentice Hall.

McCalley, R. W. (1992). Marketing channel development and management. New York, NY: Quorum Books.

Mendelson, H., \& Pillai, R. R. (1998). Clockspeed and information response: Evidence from the information technology industry. Information Systems Research, 9(4), 415-433. http://dx.doi.org/10.1287/isre.9.4.415

Mendelson, H., \& Whang, S. (2000). Introduction to the special issue on the information technology industry. Management Science, 46(4), 1-3. http://dx.doi.org/10.1287/mnsc.46.4.i

Reibstein, D. J., \& Farris, P. W. (1995). Market share and distribution: A generalization, a speculation, and some implications. Marketing Science, 14(3), 190-202. http://dx.doi.org/10.1287/mksc.14.3.G190

Zhou, N., Zhuang, G., \& Yip, L. S. C. (2007). Perceptual difference of dependence and its impact on conflict in marketing channels in China: An empirical study with two-sided data. Industrial Marketing Management, $36,309-321$. 